\title{
Identification of nonlinear-nonlinear neuron models and stimulus decoding
}

\author{
Aurel A Lazar, Yevgeniy B Slutskiy \\ From Twenty Second Annual Computational Neuroscience Meeting: CNS*2013 \\ Paris, France. 13-18 July 2013
}

The majority of neural encoding models employed today consist of a linear feature-selection stage followed by a static memoryless nonlinearity generating the neuronal rate of response. Although such linear-nonlinear $(\mathrm{LN})$ models have been proven useful in characterizing computation in many neurons (see [1] and references therein), they exclude the biophysics of action potential generation and, with a few exceptions [2-4], are limited to describing only linear computations. A large body of evidence [5] suggests that various synaptic microcircuits and complex dendritic morphologies exhibit a rich repertoire of nonlinear computations on a single neuron level. Importantly, such computations are observed not only for individual inputs [2,3] but also between inputs, as manifested by multiplicative interactions between them [5]. At the same time, the highly nonlinear nature of action potential generation has been shown to adversely affect many existing identification methodologies and typically presents itself as artifactual stimulus processing $[3,6]$.

Here we investigate nonlinear-nonlinear neuron models that combine both biophysics and dendritic computation. Specifically, we consider models in which the nonlinear dendritic processing of input stimuli is described by a Volterra series [4] and the aggregate dendritic current is encoded into a sequence of action potentials by a biophysical conductance-based model. We first present a novel experimental approach for characterizing the biophysical spike generator using conditional phase response curves [7]. We then prove that only a projection of Volterra kernels onto the space of input signals can be identified and present an efficient algorithm for estimating these projections. The identification is robust in the presence of noise, scales favorably with the order of nonlinear interactions and affords high-resolution identification due to a

\footnotetext{
* Correspondence: aurel@ee.columbia.edu

Department of Electrical Engineering, Columbia University, New York, NY, 10027, USA
}

(c) 2013 Lazar and Slutskiy; licensee BioMed Central Ltd. This is an Open Access article distributed under the terms of the Creative Commons Attribution License (http://creativecommons.org/licenses/by/2.0), which permits unrestricted use, distribution, and reproduction in any medium, provided the original work is properly cited. basis decomposition of the projections. Furthermore, either synthetic or naturalistic stimuli can be used to identify the model. Finally, we consider a nonlinear-nonlinear population encoding scheme and construct a nonlinear decoder that is capable of reconstructing the original stimulus directly from spike times. This should be contrasted with linear decoders applied to firing rates of LN models $[8,9]$. To the best of our knowledge, this is the first demonstration of a nonlinear decoder capable of high-fidelity reconstruction from a single multidimensional spike train in the nonlinear-nonlinear setting.

\section{Acknowledgements} \#FA9550-12-1-0232 and in part by NIH under grant \#R021 DC012440001.

Published: 8 July 2013

\section{References}

1. Wu MC-K, David SV, Gallant JL: Complete functional characterization of sensory neurons by system identification. Annual Reviews of Neuroscience 2006, , 29: 477-505.

2. Sharpee T, Rust NC, Bialek W: Analyzing Neural Responses to Natural Signals: Maximally Informative Dimensions. Neural Computation 2004, 16: $223-250$.

3. Schwartz O, Pillow JW, Rust NC, Simoncelli E: Spike-triggered neural characterization. Journal of Vision 2006, 6:484-507.

4. Marmarelis VZ: Nonlinear Dynamic Modeling of Physiological Systems. Wiley-IEEE Press 2004.

5. Koch C: Biophysics of Computation: Information Processing in Single Neurons. Oxford University Press 1999.

6. Jonathan WPillow, Eero PSimoncelli: Biases in white noise analysis due to non-Poisson spike generation. Neurocomputing 2003, , 52-54: 109-115.

7. Kim AJ, Lazar AA: Recovery of Stimuli Encoded with a Hodgkin-Huxley Neuron Using Conditional PRCs. Phase Response Curves in Neuroscience Springer; 2011 .

8. Stanley GB, Li FF, Dan Y: Reconstruction of natural scenes from ensemble responses in the lateral geniculate nucleus. J Neurosci 1999, , 19: 8036-8042. population of retinal ganglion cells. J Neurophysiol 1997, 78(5):2336-2350.

doi:10.1186/1471-2202-14-S1-P367

Cite this article as: Lazar and Slutskiy: Identification of nonlinear-

nonlinear neuron models and stimulus decoding. BMC Neuroscience 2013 14(Suppl 1):P367.
The research reported here was supported in part by AFOSR under grant

9. Warland DK, Reinagel P, Meister M: Decoding visual information from a 